\title{
JET BREAKUP and SPRAY FORMATION in a DIESEL ENGINE
}

\author{
James Glimm \\ Department of Applied Mathematics and Statistics - \\ University at Stony Brook, Stony Brook, NY 11794-3600, USA and \\ Center for Data Intensive Computing, \\ Brookhaven National Laboratory, Upton, NY 11973-5000, USA \\ Xiaolin Li \\ Department of Applied Mathematics and Statistics \\ University at Stony Brook, Stony Brook, NY 11794-3600, USA \\ Myoung-Nyoun Kim, Wonho Oh, Andréa Marchese and Roman Samulyak \\ Center for Data Intensive Computing, \\ Brookhaven National Laboratory, Upton, NY 11973-5000, USA \\ Constantine Tzanos \\ Reactor Analysis and Engineering, \\ Argonne National Laboratory, Argonne, $\amalg$ 60493, USA
}

\begin{abstract}
The breakup of injected thel into spray is of key interest to the design of a fuel efficient, nonpolluting diesel engine. We report preliminary progxess on the numerical simulation of diesel fuel injection spray with the front tracking code FronTier. Our simulation design is set to match experiments at ANL, and our present agreement is semi-quantitative. Future efforts will include mesh refinement studies, which will better model the turbulent flow.
\end{abstract}

\section{Keywords}

Diesel spray, multiphase flow, turbulence, combustion, front tracking, cavitation, breakup, droplet, jet speed, phase transition.

\section{Tntroduction}

The design of a fuel efficient, nonpolluting diesel engine is the subject of intensive international research efforts. The breakup of the injected jet of diesel fuel into spray is of key interest to such investigations because much of the pollution comes from unburnt fuel deposited on the walls of the engine chamber, and because control of the spray drcplet size can mitigate this effect. Spray formation is sensitive to many details of the injection system design, including the driving pressure in the nozzle, the nozzle shape, and the flow turbulence and phase transitions in the nozzle.

For the above reasons, a simulation study of the breakup of the diesel jet into spray is of considerable interest. Factors complicating such a study include

1. The stiff equation of state (EOS) for the liquid diesel fuel,

2. Presence of shock waves or near shock waves and phase transitions in the flow field,

3. The multiscale nature of the flow field, especially regarding turbulence in the nozzle and the multiscale breakup of the jet irto droplets and spray (droplets are about 10 microns in size),

4. Sharp boundaries for the liquid diesel fuel - ambient chamber gas interface during the breakup period.

In this paper we report preliminary progress on the numerical modeling of the jet breakup, based on the front tracking code FronTier. 


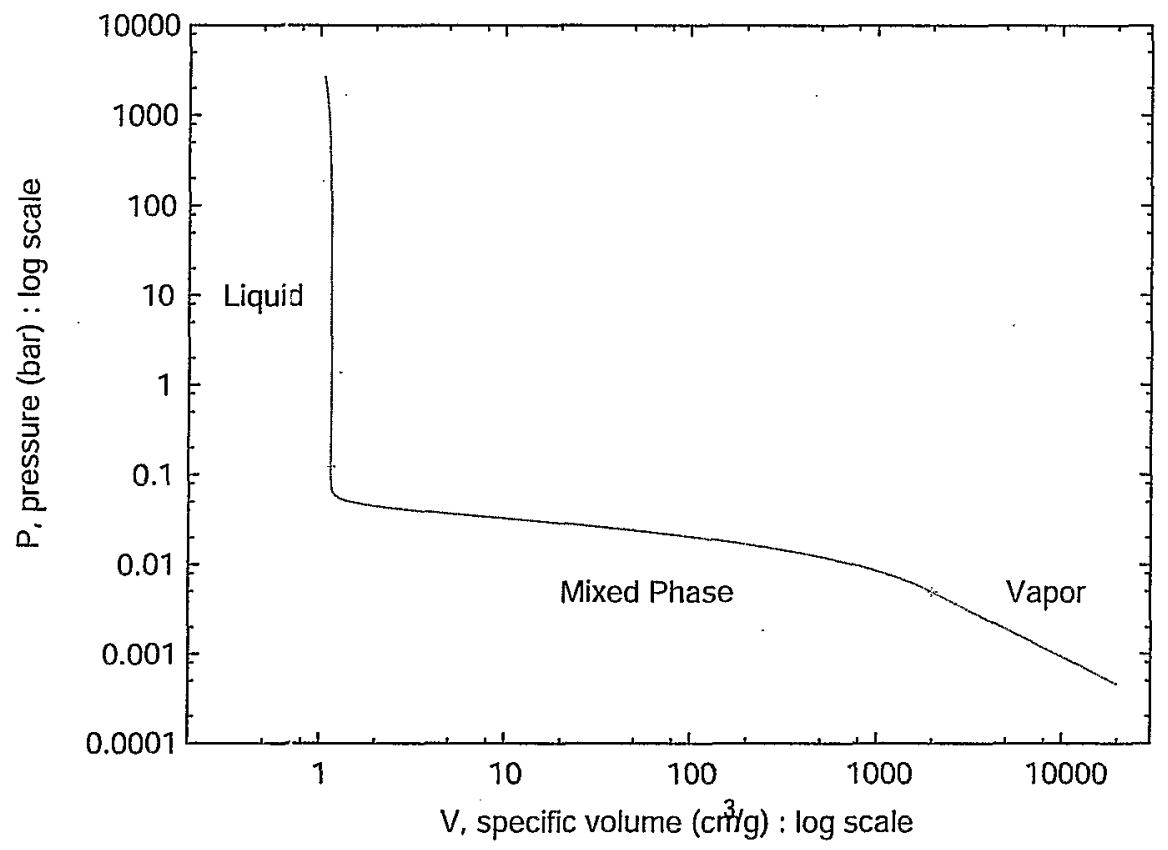

Figure 1: $P$ vs. $V$ isentrope for diesel fuel.

\section{$2 \quad$ Numerical Modeling}

We model the injection as $2 \mathrm{D}$ axisymmetric, and we refer the reader to [1,2] for a detajled description of FronTier front tracking code. We mention here some recent additions to the code for this project.

The diesel fuel is modeled as isentropic, with an analytic EOS. To incorporate the cavitation (vaporization) of the fuel in the nozzle, we mordel the fuel piecewise as follows. The EOS is given by a gamma law gas in the vapor region, by a stiffened gamma law gas in the liquid region, and by the homogeneous equilibrium model (see [7]) along an isentrope in the mixed region (where vapor and liquid co-exist). $P$ vs. $V$ is graphed in Fig. 1.

Because diesel fuel is slightly viscous, viscosity has been added to our code resulting in an explicit NavierStokes solution algorithm. To control stability, a parabolic CFL time step control was also added. For the presently considered meshes, the parabolic time step control is nearly the same as the hyperbolic CFL constraint required for the Euler (hyperbolic) terms in the Navier Stokes equation. As the mesh is refined in future studies, the parabolic time step control will become limiting. Accordingly, we will first subcycle, and take smaller time steps in the parabolic terms alone, and with further mesh refinement, we will switch to a partly implicit algorithm.

Slip and no-slip boundary conditions have been used in the fuel container and nozzle. More general possibilities will be considered in later stages of this work.

To model the high pressure imposed on the fuel, we introduce a time dependent fixed pressure (Dirichlet boundary) condition at the inlet of the injector's fuel container. For subsonic isentropic flow, this prescribed pressure at the computational boundary gives complete boundary conditions.

Along the fuel - chamber gas interface, the computational frame of reference is adjusted at each time step to allow the interface points to move in Lagrangian frame with the moving fluid. Tangential interface motion is an isomorphism of the interface. Thus an arbitrary tangential motion is, in principle, allowed. The purpose of using the moving frame is to choose this tangential motion optimally to reduce remeshing and numerical surface tension effects which inhibit Kelvin-Helmholtz roll-up effects on the surface of the jet. The frame is treated as follows. 


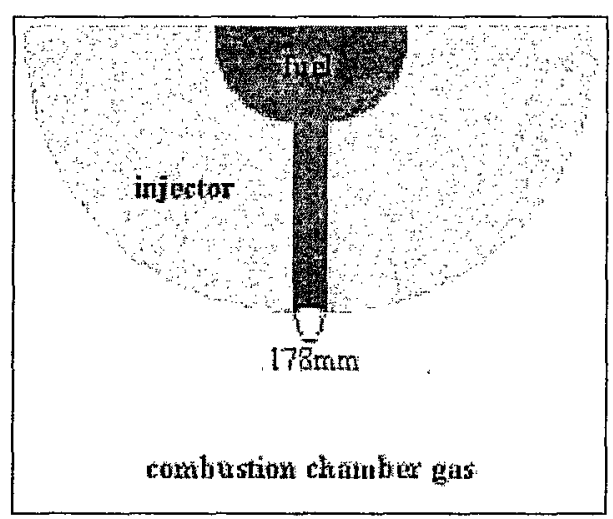

Figure 2: Geometry of injection reservoir and nozzle leading to combustion chamber.

For each point $p$ on the interface curve,

1. Calculate the frame velocity at $p$ as the density-weighted average of velocities:

$$
V=\frac{\rho_{\text {fuel }} V_{\text {fuel }}+\rho_{\text {gas }} V_{\text {gas }}}{\rho_{\text {fuel }}+\rho_{\text {gas }}}
$$

where the subscripts fuel and gas refer to the two states at point $\mathrm{p}$.

2. Subtract the frame velocity from the states used to propagate the point $p$,

3. Perform the normal and tangential computations to update the states at $p$,

4. Add the frame velocity to the computed states,

5. Propagate the point $\mathrm{p}$, using the frame velocity plus the normal velocity computed in step 3 .

The use of a front tracking colle eliminates numerical mass diffusion and thus treats the complication associated with the sharp interface boundary. Our future plans are to use automatic mesh refinement to address the multiscale issues.

\section{ANL Experiment}

Fig. 2 displays a detail in the experimental setup at ANL [3]. A finite pulse of diesel fuel is injected at a pressure of 500 bar into a chamber of SF6 (a heavy, inert gas chosen to simulate the compressed air in a diesel engine).

Using synchrotron x-ray imaging, ANL scientists have measured the mass on line segments passing through the jet in both axial and radial directions. From this data they also note the velocities of the tip and the tail of the fuel jet as it enters the combustion chamber $[3,5,4,6]$. We have installed into our code diagnostics to record similar data in our simulations.

\section{Preliminary Results}

Fig. 3 shows the density of the diesel fuel in our latest Fron Tier run. As expected, the simulation shows the fuel cavitation at the nozzle inlet and continued phase transitions throughout the body of the jet. 

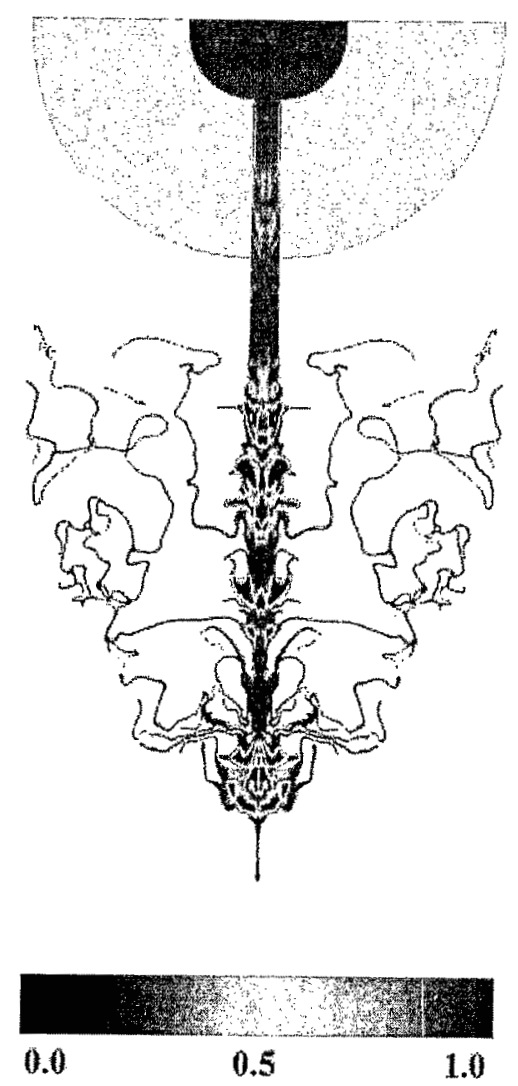

Figure 3: I)ensity of diesel fuel as computed by FronTier. 
The following fuel parameters are important for prediction: viscosity for pure liquid, sound speed for pure liquid, density of saturated liquid, pressure of saturated liquid, gamma for pure vapor, density of saturated vapor, and rise time of pressure pulse. With variations in these parameters we see different amounts of entrainment and different jet velocities. We are currently determining the set of parameters to best model the diesel \#2 used in the ANL experiment.

\section{Conclusion, Future Work}

Our present agreement with experiment is semi-quantitative. Some parameters not measured experimentally but important for simulction have been discovered. Parameter and mesh refinement studies are planned for future efforts. Our studies will include the following four aspects:

1. The initial pressure build-up of the fuel container (wave reflections and transmissions),

2. The response of the jet valocity to the applied pressure in the fuel container,

3. Cavitation, one factor in formation of the spray,

4. Entrainment, another factor for the formation of the spray.

Acknowledgement: USDOE

\section{References}

[1] J. Glimm, J. W. Grove, X.L. Li, K.-M. Shyue, Q. Zhang, and Y. Zeng. Three dimensional front tracking. SIAM J. Sci. Cor:ap., 19:703-727, 1998.

[2] J. Glimm, J. W. Grove, X.-L. Li, and D. C. Tan. Robust computational algorithms for dynamic interface tracking in three dimensions. SIAM J. Sci. Comp., 21:2240-2256, 2000.

[3] Andrew G. MacPhee, Mark W. Tate, and Christopher F. Powell. X-ray imaging of shock waves generated by high-pressure fuel sprays. Science, 295:1261-1263, 2002.

[4] C. F. Powel, Y. Yue, Sreenuth Gupta, Armon McPherson, R. Poola, and J. Wang. Development of a quantitative measurement of a diesel spray core using synchrotron x-rays. In Eighth International Conference on Liquid Atomization and Spray Systems, Pasadena, CA, 2000.

[5] C. F. Powel, Y. Yue, R. Poola, and J. Wang. Time resolved measurements of supersonic fuel sprays using synchrotron x-rays. J. Synchrotron Rad., 7:356-360, 2000.

[6] C. F. Powel, Y. Yue, R. Poola, J. Wang, M. Lai, and J. Schaller. Quantitative x-ray measurements of a diesel spray core. In Proc. 14th Annual Conference on Liquid Atomization and Spray Systems (ILASS), Dearborn, MI, 2001.

[7] G. Wallis. One-Dimensiona! Two-Phase Flow. McGraw-Hill, New York, 1969. 